\title{
The Analytical Aspects and Regulatory Challenges of Biomarker Discovery: Examples from the Field of Neurodegeneration
}

\author{
Robert L. Martone • Richard Snyder • Arron S. L. Xu • \\ Rachel Y. Reams
}

Published online: 18 April 2012

(C) Springer Science+Business Media, LLC 2012

\begin{abstract}
Drug discovery is guided by theories regarding disease pathogenesis, and biomarkers are the essential analytical tools used to translate those theories into clinical experiments. Biomarkers can be used to expedite clinical trials by permitting selection of the most uniform and responsive patient population, by reporting on target engagement of a particular therapeutic mechanism, and by permitting measurement of biochemical or physiological correlates of disease mechanism with end points that may be more sensitive, immediate, or statistically robust than phenotypic or behavioral changes. Biomarker discovery necessarily begins with an analytically validated assay for an analyte, which is later qualified by demonstrating its utility in some context of disease and therapeutic state. Ultimately, just as with the drug discovery process as a whole, successful biomarker discovery and development depends upon a clear understanding of the disease process. This article reviews the challenges of biomarker discovery with an emphasis on fluid biomarkers in neurodegeneration.
\end{abstract}

Keywords Validation - Analytical · Biomarker . Qualification · Analyte $\cdot$ Matrix $\cdot$ Accuracy $\cdot$ Precision .

Sensitivity $\cdot$ Linearity $\cdot$ Immunoassay $\cdot$ Amyloid beta $\cdot A \beta$. Microtubule-associated protein tau $\cdot \mathrm{MAPT} \cdot \alpha$-synuclein . SNCA

\section{Introduction}

Biomarker discovery is an integral component of the drug discovery process. In a well-planned discovery program,

R. L. Martone $(\varangle) \cdot$ R. Snyder · A. S. L. Xu • R. Y. Reams

Biomarker Center of Excellence,

Covance Inc., 671 South Meridian Road,

Greenfield, IN 46140, USA

e-mail: Robert.Martone@Covance.com biomarker discovery efforts commence early and proceed in parallel with the drug discovery process [1]. Efficacy biomarkers are as necessary for lead optimization and preclinical efficacy studies as they are for clinical studies, and the early incorporation of a biomarker strategy in the drug discovery process allows for adequate exploration of the role of a given analyte in disease pathogenesis, as well as its half-life, distribution, metabolism, and the possible role of post-translational modifications [2]. Biomarkers and therapeutic targets are often identified as components of hallmark pathological lesions, especially in neurodegenerative diseases, and often therapeutic target and biomarker are one and the same analyte. Likewise, genetic predisposition to a disease process informs the selection of therapeutic targets as well as diagnostic and/or stratification biomarkers.

Because biomarkers fill multiple roles in experimental pharmacology, an essential first step in biomarker discovery is to delineate the role a specific biomarker will play in the spectrum of research. The requirements for a diagnostic biomarker, for example, may be very different than those of a biomarker of disease progression or of toxicity. Biomarker applications include diagnosis or assessment of disease susceptibility, patient stratification, therapeutic target engagement, and disease progression or staging, as well as off-target and toxic effects. For a biomarker to have utility it must be informative with respect to one of these applications, and it must have an analytically validated assay that can be reproduced between different studies and different laboratories. The availability of such an assay enables the subsequent qualification of the biomarker, which is the demonstration of a disease-related change in the biomarker levels of an appropriate analyte. The relevance of the biomarker needs to be verified by additional studies, preferably conducted by different laboratories, before clinical validation and commercialization of the biomarker assay may begin (see Fig. 1). 
Fig. 1 Biomarker discovery and validation pipeline. Biomarkers having a specific application are assessed by assays that have undergone a rigorous analytical validation. Multiple parameters are evaluated according to a method validation plan. The impact of pre-analytical factors is also determined. The analytically validated assay is used to qualify the biomarker in a given disease state. After replication in additional studies in additional laboratories, the assay is optimized and commercialized $M O A$ mechanism of action

\begin{tabular}{|c|c|c|}
\hline $\begin{array}{l}\text { Specify } \\
\text { Purpose: }\end{array}$ & $\begin{array}{l}\text { Analytical } \\
\text { Validation: }\end{array}$ & Qualification \\
\hline $\begin{array}{l}\text { Interrogate MOA } \\
\text { Efficacy } \\
\text { Stratification/Risk } \\
\text { Diagnostic }\end{array}$ & \multirow{5}{*}{$\begin{array}{l}\text { Method Validation Plan } \\
\text { Linearity/ } \\
\text { Measurement Range } \\
\text { Precision (Replication) } \\
\text { Accuracy/Recovery } \\
\text { Sensitivity } \\
\text { Analytical Specificity } \\
\text { (Interference) } \\
\text { Dilution Verification } \\
\text { Reference Interval } \\
\text { (Normal Range) } \\
\text { Stability } \\
\text { Specimen Carryover } \\
\text { Analytical Stability Period } \\
\text { (Length of Run) } \\
\text { Statement of Claims } \\
\text { Summary Report }\end{array}$} & $\begin{array}{l}\text { Analyte is representative of } \\
\text { disease state, MOA, etc. }\end{array}$ \\
\hline $\begin{array}{l}\text { Staging } \\
\text { Toxicity }\end{array}$ & & Replication \\
\hline Pre-Analytical & & $\begin{array}{l}\text { Multiple laboratories reproduc } \\
\text { study results with assay }\end{array}$ \\
\hline $\begin{array}{l}\text { Factors: } \\
\text { Sample Type } \\
\text { Stability }\end{array}$ & & \\
\hline $\begin{array}{l}\text { Circadian Rhythm } \\
\text { Sample Handling } \\
\text { Sample Additives }\end{array}$ & & $\begin{array}{l}\text { Assay Optimization } \\
\text { \& Commercialization }\end{array}$ \\
\hline
\end{tabular}

\section{The Challenges of Biomarker Discovery}

Biomarkers may be identified by virtue of their presence in pathological disease-associated lesions, such as the components of the senile plaques, congophilic angiopathy, and neurofibrillary tangles that are characteristic of Alzheimer's disease (AD) [3] or the Lewy Bodies that are pathognomic for Parkinson's disease (PD [4]). This is the source of interest in the pathogenic fragment of the amyloid precursor protein (APP), amyloid beta (A $\beta)$, the microtubuleassociated protein (MAPT) tau, and $\alpha$-synuclein (SNCA), respectively. The association of a biomarker with these lesions carries with it significant face validity; however, this association may be due to secondary processes or epiphenomena, and a functional relationship with disease must always be established.

Alternatively, biomarkers may be identified in a hypothesisfree manner by surveying total proteins present in appropriate samples using proteomic approaches [5], assessment of global post-translationally modified proteins (eg, phosphoproteomics [6]) and via prediction from genomics approaches. The global proteomics approach has proven most powerful in the identification of multiple analyte panels (MAPs) of several analytes, which collectively provide a significantly more robust predictive value than any of the constituent analytes provide individually [7]. Expression profiling may yield clues regarding differential expression of genes and perturbations of signaling networks in disease states or in response to drug treatments [8]. Surrogate biomarkers associated with a disease state or a therapeutic effect also may be discovered through unbiased expression profiling. Genome-wide association studies (GWAS) have proven increasingly valuable in linking disease with genotypic variation (eg, the sequence variants associated with genetic risk for PD [9]). Other promising new genomics approaches include analysis of regulatory micro RNAs (miRNAs) $[10 \bullet, 11]$ as well as examination of the genome-wide splice array $[8,12 \cdot]$.
Biomarker analytes may be restricted to specific compartments, such as the plasma compartment or the cerebrospinal fluid (CSF). This is especially important for most central nervous system (CNS) studies, where the matrix for biochemical biomarkers is often restricted to lumbar or, rarely, ventricular CSF samples, because biomarkers such as tau are often not detectable in plasma serum. There are multiple factors that may impact analyte levels in CSF or other matrices that are independent of disease process, including transport across compartment barriers, such as the bloodbrain barrier (BBB), CSF circulation to point of collection, and analyte stability. Analyte levels also may be responsive to biological activity, such as neuronal activity in the brain, and may exhibit circadian patterns or gender specificity.

Given these complexities, CNS diseases often require a biomarker approach examining a surrogate target tissue. For example, biopsies of peripheral nerve may serve as a surrogate for CNS neuronal pathophysiology. Biopsies of cutaneous nerves [13], enteric nerves [14] and the nerve supply of salivary glands [15] are being investigated for their utility for the investigation of the intraneuronal pathology that accompanies disease progression in PD. Surrogate biomarkers by definition are a step removed from the site of action and/or desired clinical endpoint, and therefore may carry with them unique qualification concerns [16].

\section{Analytical Validation}

A robust, analytically validated assay is necessary for a clear understanding of an analyte's utility as a biomarker. The analytical validation of an assay is a close examination of the physiochemical properties of the analyte being measured, the analyte's interaction with other assay components, and the inherent platform sensitivity. Validation parameters interrogate an analyte's stability, its interaction 
with matrix components, and the range of its levels in relevant matrices and populations in the context of the robustness of an assay detection technology. Here, we review approaches for soluble biomarkers, using ligandbinding approaches such as enzyme-linked immunosorbent assay (ELISA). Other biomarker modalities such as imaging have their own complex validation challenges [17], which are beyond the scope of this review, as are the issues confronting the validation of bioanalytical methods for small molecules [18] and large molecule therapeutics [19]. The American Association of Pharmaceutical Scientists (AAPS)/ U.S. Food and Drug Administration (FDA) 3rd Bioanalytical Workshop Best Practices for quantitative bioanalytical methods validation [20], as well as best practices concerning characterization of assay reagents, standards, quality controls, study samples, and analyte stability have been reported [21]. Recommendations of the European Medicines Agency (EMEA) are available [22]. Recommendations reflecting a pharmaceutical industry perspective emphasize the importance of immunoassay specificity, since the analyte is not typically extracted from matrix before assessment in this type of assay [23]. Others emphasize the importance of parallelism among assay validation parameters [24]. Validation of a clinical diagnostic biomarker and its analytical assays often requires additional criteria such as patient population criteria and clinical reference range

The degree to which an assay is analytically evaluated depends upon the assay's intended use, and will determine the validation methodology employed. It is often the case that a biomarker assay intended only for exploratory use will be minimally validated compared to an assay intended for clinical use. Research Use-Only applications (RUO) are outside the umbrella of guidance provided by such governing bodies as the FDA and EMEA. Therefore, despite the extensive literature on the topic of immunoassay validation schemes, the make-up of the experimental plans used to validate an immunoassay for a given intended use varies greatly between research institutions.

The parameters evaluated during an analytical validation of an assay as well as the AAPS recommended acceptable values have been reported [25]. In general terms, these parameters evaluate proportionality between input levels of analyte and assay signal which, in a typical sandwich ELISA assay, reflect upon the capture capacity and signal/ noise ratio (sensitivity, linearity, reference interval), the interaction of the analyte with matrix components (accuracy/ recovery, dilutional specificity), intra- and interassay precision and drift (influenced by manufacturing factors such as capture antibody coating or conjugation technique, and the robustness of the assay protocol with respect to pipetting technique, washing methods, as well as mixing and incubation time and temperature), and the stability of the analyte, which may relate to an analyte's degradation or aggregation (evaluated in a stability experiment). An assay's reproducibility and face validity (method comparison) are also evaluated (see Table 1).

Accuracy is often assessed by simple spike-recovery experiments using a concentrated and well-quantified source of analyte and a pooled matrix to create spikes that span the standard curve range. In general, the final volume of the spike should be no more than $10 \%$ of the final total sample volume, and the common acceptance criteria is recovery of $80 \%$ to $120 \%$ of the nominal value [20]. In cases where the endogenous analyte level is within the limit of quantitation of the assay, the matrix should be analyzed alongside the spiked samples to allow for correction of the measured spike values with that of the measured endogenous analyte value. Ideally, spikes should be at least three times the endogenous analyte level because the formulas used for spike-recovery with endogenous correction are vulnerable to situations in which the spike value is very near the endogenous value. Such experiments are confounded by the existence of matrix proteins that bind to the analyte, and thus, cause underrecovery of analyte.

The complexity of the matrix fluid often complicates a biomarker assay with respect to the nonspecific matrix interference for analyte detection. Poor sensitivity or linear range may be attributable to poor performance of the detection reagents, signal quenching, or other signal interference from the matrix. Many alternative platforms are available for the enhancement of the sensitivity and linear range of protein assays. Among the microplate-based immunochemical assays, options beyond the basic enzyme-conjugated colorimetric or fluorometric assay include electrochemiluminescent assays [26, 27], solution ELISA [28], AlphaLISA (PerkinElmer, Waltham, MA) [29], TR-FRET (time-resolved fluorescence [TRF]/fluorescence resonance energy transfer), dendrimers [30], and proximity ligation assays [31]. There are several studies comparing the relative utilities of various assay platforms [27, 28, 32]; however, it has been reported that it is difficult to predict the best platform for a given application, and that the best platform often needs to be selected empirically [33]. Certain detection modalities such as luminescence and TRF perform quite differently depending upon the instrumentation used to analyze the signal, and therefore, assays based upon them can exhibit large differences in apparent sensitivity between labs.

Because the interaction of matrix components with the analyte or with assay components can cause significant problems with an assay, a number of analytical strategies have been employed to address this challenge. Sample dilution may reduce nonspecific matrix interference for biomarker detection, but the analytical sensitivity of a biomarker assay and the low endogenous abundance of a biomarker in biological fluids may preclude this strategy for 
Table 1 Analytical validation parameters: challenges and guidance on acceptance criteria*

\begin{tabular}{|c|c|c|}
\hline Analytical parameter & Challenges & Guidance on acceptance criteria \\
\hline Standard curve performance & $\begin{array}{l}\text { Choosing the appropriate model (eg, 4-PL, 5-PL) } \\
\text { or weighting scheme }\end{array}$ & $\begin{array}{l}\leq 20 \% \mathrm{RE} \text { vs nominal value for each standard } \\
\text { point, } \leq 15 \% \mathrm{CV} \text { for replicates }\end{array}$ \\
\hline $\begin{array}{l}\text { Linearity/measurement } \\
\text { range }\end{array}$ & $\begin{array}{l}\text { Assay capture antibody capacity, platform/detection } \\
\text { modality (eg, TRF vs absorbance) }\end{array}$ & $\begin{array}{l}\text { Accuracy and precision of fractionally diluted } \\
\text { samples that span the range of the standard } \\
\text { curve and have the \%matrix equal to the } \\
\text { MRD should } \leq 20 \% \mathrm{RE} \text { and } \% \mathrm{CV}\end{array}$ \\
\hline Accuracy/recovery & $\begin{array}{l}\text { Matrix interaction (eg, analyte-binding proteins } \\
\text { present in matrix that mask capture/detection epitopes) }\end{array}$ & $\begin{array}{l}\leq 20 \% \text { RE. Spike should be no more than } 5 \% \text { of } \\
\text { the total sample volume. When endogenous } \\
\text { protein is measurable, this value should be used } \\
\text { to correct the nominal value of the spiked sample }\end{array}$ \\
\hline $\begin{array}{l}\text { Sensitivity (lower limit } \\
\text { of quantitation) }\end{array}$ & $\begin{array}{l}\text { Capture/detection antibody performance, blocking } \\
\text { efficiency, platform, signal quenching, interference }\end{array}$ & $\begin{array}{l}\text { LLOQ set at lowest sample where the } \\
\text { interbatch } \% \mathrm{CV} \text { and } \% \mathrm{RE} \text { are both } 25 \%\end{array}$ \\
\hline Dilutional linearity & $\begin{array}{l}\text { Matrix interaction, sample dilution buffer performance, } \\
\text { prozone (hook effect [eg, capture antibody capacity]) }\end{array}$ & $\begin{array}{l}\text { The maximum dilution allowable in a beyond the } \\
\text { MRD is set at the last dilution factor in a series } \\
\text { of dilutions at which the } \% \mathrm{RE} \text { is } \leq 20 \%\end{array}$ \\
\hline Intra-/interassay precision & $\begin{array}{l}\text { Assay manufacture (eg, capture antibody adherence to } \\
\text { solid support), robustness (eg, mixing parameters, } \\
\text { incubation time/temp), matrix interaction }\end{array}$ & Both intra- and interbatch precision are $\leq 20 \% \mathrm{CV}$ \\
\hline Drift/edge effects & $\begin{array}{l}\text { Assay manufacture, robustness (eg, mixing } \\
\text { parameters, incubation time/temp) }\end{array}$ & $\begin{array}{l}\text { Patterns in signal intensity based on plate location } \\
\text { should not be observable [98] }\end{array}$ \\
\hline Reference interval & $\begin{array}{l}\text { Assay capture capacity, matrix interaction, pre-analytical } \\
\text { factors (eg, sample collection/storage methods) }\end{array}$ & $\begin{array}{l}\text { The frequency distribution of the reference interval } \\
\text { should not contain the LLOQ or ULOQ of the assay }\end{array}$ \\
\hline Stability & $\begin{array}{l}\text { Analyte stability (eg, vulnerability to serum } \\
\text { proteases)/aggregation }\end{array}$ & $\begin{array}{l}\text { Analyte within matrix should exhibit } \leq 20 \% \mathrm{RE} \\
\text { compared to the nominal value when stored in } \\
\text { a manner equivalent to how the study samples } \\
\text { will be handled }\end{array}$ \\
\hline Specificity & Antibody performance, matrix interaction & $\begin{array}{l}\text { Increase in signal should not be observed from } \\
\text { addition of proteins similar to the analyte of interest }\end{array}$ \\
\hline Method comparison & Robustness, instrument calibration & 4-6-30 Rule ${ }^{\mathrm{a}}$ \\
\hline
\end{tabular}

4-PL four parameter logistic; 5-PL five parameter logistic; TRF time-resolved fluorescence; $\% R E$ percentage relative error; $\% C V$ percentage coefficient of variation; $M R D$ minimum required dilution; $L L O Q$ lower limit of quantitation; temp temperature; ULOQ upper limit of quantitation; AAPS American Association of Pharmaceutical Scientists

* Parameters are listed with challenges often encountered during analytical validation and guidance on acceptance criteria provided by AAPS [25] except where otherwise indicated

${ }^{a}$ The 4-6-30 Rule: At least four of six assay values must be within $30 \%$ of their nominal concentrations

reduction of matrix interference. In contrast, if analyte levels are exceedingly low, samples may be concentrated or multiple sample volumes may be loaded to a solid phase assay in an effort to saturate capture reagents with analyte from a larger total sample volume. Often, detergents, chelators, or chaotropes are employed to reduce nonspecific protein-protein interactions that may contribute to matrix effects. In utilizing such techniques, the investigator should remain cognizant of the fact that antibody-analyte interaction is also a protein-protein interaction susceptible to the same treatments, even though their interaction (binding) is generally of greater affinity. An additional confounder for immunoassays employed in drug research is interference from drug molecules that bind to the target analyte, and thus, potentially obscure important epitopes required for assay antibody binding. The standard methodology to reduce this binding (eg, acidification of the sample) also can suppress assay antibody binding, or increase matrix interference from denatured matrix proteins. Binding equilibrium experiments also must be performed to show that the capture antibodyanalyte complex is able to reach a steady state under the lower $\mathrm{pH}$ conditions during the allotted incubation time.

Pre-analytical factors may contribute to both matrix effects and analyte stability, and appropriate sample collection protocols need to be delineated, including need for anticoagulants/ chelators, addition of enzyme inhibitors, collection tube material (eg, plastic, glass), sample preparation (centrifugation, denaturation), freeze-thaw cycle, and storage conditions. Disease state and physical condition may impact samples as well. It is important that clear instructions be given to surgical staff when collecting samples from patients or research animals. Training procedures must be followed to ensure consistency in sample collection (eg, collection protocol, sample containers, sample preparation and storage). 
The preparation, handling, and quality control of standard reference material used in standard curves are critical to the successful execution of an assay. Biological control standard materials may be hydrophobic or have a propensity to aggregate, precipitate, or degrade. Denaturation, oxidation, and reduction of protein standards used in immunochemical assays of biomarkers are particularly problematic, and can easily led to failure of an analytical validation or sample analysis. It is of utmost importance for a biomarker assay meant for large-scale use that the standard and control material be formulated in such a way that the materials are amenable for assay scale-up, stable for long periods of time, and exhibit excellent lot-to-lot consistency with respect to the signal obtained from the intended assay. If standard material cannot be made in large, stable, reproducible batches, extreme caution must be exercised when comparing and interpreting biomarker data obtained when different batches of control standard materials are used. This caveat is also applicable to other rare assay components such as the assay antibodies, enzymes, and other signal detection reagents that are part of the biomarker detection assay. Quality control of the control standards may encompass, but is not limited to, molecular characterization (eg, proteins and small molecule biomarker standards), activity analysis (eg, enzymatic activity assay standards), or other functional analysis (eg, standards for cell-based in vitro activity assays). Reference control standards of assays must be analyzed and quantified by an analysis independent of the biomarker assay in analytical validation or sample analysis. Manufacturers of such assays should ideally align future assay production lots to the results obtained by a master lot. Assay comparison should minimally reveal congruence in relative values of samples, if not agreement of absolute calculated values. Assays that require standard material are beholden to other orthogonal technologies that can accurately quantify the standard material before it is used in an assay. Absence of techniques that can quantify the standard material before assay implementation may limit the utility of such a biomarker assay to determination of relative values (eg, antibody titer assay). The World Health Organization (WHO) can be a source of reference standard proteins that can be used to compare the results of different immunoassays. Difference in absolute values may result from difference in antibody affinities or epitopes, differences in sensitivities of assay platforms (if applicable), or handling of reagent components critical to the creation of the assay standard curve.

\section{Examples of Analytical Validation Challenges}

Some of the most intensely studied CSF analytes are illustrative of many of the issues encountered during the analytical validation of a biomarker. Here, we will review efforts to validate assays for the $\mathrm{AD}$-associated $\mathrm{A} \beta_{42}$, tau (total tau [t-tau]) and phospho-tau (p-tau), use of which has been recommended for $\mathrm{AD}$ clinical trials [34], as well as the PD-associated protein SNCA (see Table 2).

$A \beta$ is a secreted peptide with multiple genetic linkages to $\mathrm{AD}$ both regarding its precursor protein (APP) and components of the enzyme complexes responsible for generation of $\mathrm{A} \beta$ from APP (the presenilins, PS1, and PS2) [3]. A $\beta$ is also widely regarded as forming a pathogenic oligomeric species in $\mathrm{AD}$; thus, $\mathrm{A} \beta$ is implicated functionally and genetically in $\mathrm{AD}$ pathogenesis, and is regarded as a therapeutic target, as well as a diagnostic biomarker and biomarker of disease progression. Several biomarker assays using multiple different platforms have been developed to interrogate the ability of a test therapeutic compound to cause ostensibly therapeutic reductions in $A \beta$ or $A \beta$ oligomers $[35,36]$.

However, $A \beta$ is a remarkably challenging analyte. $C$ terminal heterogeneity results in peptides ranging from 38 to 43 amino acids in length. The 40 -amino acid peptide is most abundant, while the longer peptides (eg, $A \beta_{42}$ ), which are more hydrophobic and more prone to aggregation, are considered more toxic. Amino-terminal truncations and pyroglutamate modifications also occur and have been implicated in the disease process [37]. Aggregation results in the formation of insoluble amyloid fibrils as well as soluble oligomers, which are reported to be central to pathogenesis, although there is controversy regarding the specific species of oligomer involved. The poor solubility of the analyte and its propensity to aggregate necessitate very specific preanalytical handling [38,39]. The secretion of $A \beta$ is activity dependent, shows extensive fluctuations throughout the day [40], and it is actively transported across the BBB, being a substrate of transporters such as P-glycoprotein (Pgp) [41] and low density lipoprotein receptor-related protein 1 (LRP1) $[42,43]$. Indeed, decreased clearance across the BBB has been implicated in disease pathogenesis [44]. While brain $\mathrm{A} \beta_{42}$ load increases with disease, $A \beta_{42}$ measured in CSF decreases significantly as early as 10 years before onset of cognitive impairment [45]. However, turnover of $A \beta$ in humans is rapid [46], and stable isotope labeling [47] has been used to confirm the inhibition of $A \beta$ generation by compounds such as the $\gamma$-secretase inhibitor LY-450139 (Semagacestat), demonstrating that pools of $A \beta$ are dynamic and show measurable changes in response to attempts at therapeutic intervention.

In the case of LY-450139, A $\beta$ was an effective biomarker of target engagement, by demonstrating, as predicted, a compound-dependent reduction of $\mathrm{A} \beta$ generation and secretion into the CSF. However, a far more complex question is whether pharmacological manipulation of this biomarker can be correlated with changes in the most relevant trial end point, cognition. In this case, despite the close genetic and 
Table 2 Biomarkers of Alzheimer's disease and Parkinson's disease*

\begin{tabular}{|c|c|c|c|c|}
\hline Disease & Biomarker application & Biomarker modality & Biomarker & References \\
\hline \multirow[t]{8}{*}{ Alzheimer's disease } & \multirow[t]{6}{*}{ Diagnosis/progression } & \multirow[t]{3}{*}{ Fluid } & $\mathrm{A} \beta_{40}, \mathrm{~A} \beta_{42}, \mathrm{o} A \beta$ & {$[35,36,45,54-61]$} \\
\hline & & & t-tau, p181-tau & [53-61] \\
\hline & & & Multiple analyte panel & {$[7,64]$} \\
\hline & & \multirow[t]{2}{*}{ Genomic } & GWSA & {$[8,12 \bullet]$} \\
\hline & & & miRNA & {$[10 \bullet, 11]$} \\
\hline & & Imaging & Amyloid PET, FDG-PET & {$[62 \bullet \bullet]$} \\
\hline & \multirow[t]{2}{*}{ Stratification/susceptibility } & \multirow[t]{2}{*}{ Genomic } & $\begin{array}{l}\text { apoE4, PS mutations, APP } \\
\text { mutations, TOMM40, others }\end{array}$ & [3] \\
\hline & & & GWAS & {$[62 \bullet \bullet]$} \\
\hline \multirow[t]{6}{*}{ Parkinson's disease } & \multirow[t]{5}{*}{ Diagnosis/progression } & \multirow[t]{3}{*}{ Fluid } & SNCA, oSNCA, pSNCA & {$[68-70]$} \\
\hline & & & Urate & [99] \\
\hline & & & Multiple Analyte Panel & [7] \\
\hline & & Genomic & miRNA & {$[10 \bullet]$} \\
\hline & & Imaging & $\begin{array}{l}\text { FDG-PET, DAT-SPECT, VMAT } \\
\text { Binding, Fluorodopa PET, others }\end{array}$ & [99] \\
\hline & Stratification/susceptibility & Genomic & $\begin{array}{l}\text { Mutations in LRRK2, SNCA, PINK1, } \\
\text { DJ-1, UCHL1, Parkin, GBA, others }\end{array}$ & {$[4]$} \\
\hline
\end{tabular}

$A \beta$ amyloid beta; $o A \beta$ oligomeric amyloid beta; $t$-tau total tau; $p 181$-tau tau phosphorylated at threonine 181 ; GWAS genome-wide association studies; miRNA micro RNA; PET positron emission tomography; FDG-PET fluorodeoxyglucose positron emission tomography; apoE4 apolipoprotein E4; PS presenilin; APP amyloid precursor protein; TOMM40 translocase of outer mitochondrial membrane 40; SNCA $\alpha$-synuclein; $o S N C A$ oligomeric $\alpha$-synuclein; $P S N C A$ phosphorylated $\alpha$-synuclein; DAT-SPECT dopamine transporter-single photon emission computed tomography; $V M A T$ vesicular monoamine transporter; LRRK2 leucine rich repeat kinase 2; PINK1 phosphatase and tensin homolog (PTEN)-induced putative kinase-1; UCHL1 ubiquitin C-terminal hydrolase L1; GBA glucocerebrosidase

*Examples of fluid, genomic, and imaging biomarkers diagnostic biomarkers of disease progression used for diagnosis assessment of disease progression, patient stratification or assessment of disease susceptibility are provided

functional associations of $\mathrm{A} \beta$ with $\mathrm{AD}$, and despite the fact that this compound effectively reduced generation of this biomarker and therapeutic target, LY-450139 failed in phase 3 because it caused deterioration of cognitive function, precisely the opposite effect as was predicted [48].

MAPT contrasts in many ways with $A \beta$. Unlike $A \beta$, tau has not been genetically associated with $\mathrm{AD}$ (it has been associated with PD [4] as well as with frontotemporal lobar dementia [49]), although tau pathology is a hallmark of AD. Also, unlike $A \beta$, tau is primarily an intracellular protein, although recent studies demonstrate active secretion [50] of the protein. Another report confirms the presence of extracellular tau in the absence of neurodegeneration, but finds no correlation between levels of tau in brain interstitial fluid and in CSF [51]. Tau is subject to extensive post-translational modification, the most prominent being proteolytic processing, nitration, and phosphorylation [52, 53]. It is not clear whether disease-related changes in t-tau levels are due to augmented secretion or exocytosis of the protein through an active process, or due to a passive release from dead and dying neurons. Nor is it clear whether the tau fragments in CSF from $\mathrm{AD}$ patients are different those found in CSF from non-AD patients. Finally, the phosphorylated form of tau most often measured, p181-tau, may not represent the phosphorylation site that is most predictive of disease progression, or informative of disease state, but it is measured because of convention, and availability of an assay.

Therefore, these biomarkers, although linked to the disease by pathology, are subject to a multitude of posttranslational modifications, including truncations, phosphorylation, and aggregation, presenting a multiplicity of potential epitopes to follow, any of which might have more disease relevance than those epitopes currently being measured. These biomarkers are actively transported in ways that may alter measured levels of analyte, but there is no clear understanding of the link, if any, between those transport functions and disease. The value of these biomarkers would be questionable even if the analytical assays were unimpeachable, which they are not. The most commonly used assays are the Innotest assays (Innogenetics, Gent., Belgium) [54]. The Innotest assay for total tau interrogates only $15 \%$ of a highly variable molecule, while the p181 epitope, measured in the phospho-tau assay is only one of many phosphoepitopes for this protein, and the rationale for measuring it rather than other phosphoepitopes is controversial. High interlaboratory variability $[55,56]$ calls into question the reproducibility of studies using these assays and resulted in a call for standardization of pre-analytical and analytical methods [57]. Meanwhile a method comparison of the two versions of the Innotest assay (ELISA, and 
Luminex-based multiplex assays) resulted in widely divergent absolute analyte values [54]. Yet, reports claim utility for these assays, but only "when [an] astringent analytical protocol is used" [58]. Recent diagnostic recommendations from the National Institute on Aging (NIA) and the Alzheimer's Association workgroup for mild cognitive impairment state that "Considerable work is needed to validate the criteria that use biomarkers and to standardize biomarker analysis" [59], while recommendations regarding diagnosis of $\mathrm{AD}$ state that, although biomarkers might enhance specificity of the diagnosis, clinical criteria would continue as the "cornerstone of the diagnosis" [60]. A recent EMEA qualification opinion acknowledges issues regarding the reliability and complexity of the measurement methods, suggests that standardization may be an important part of resolving those issues, and while expressing concern that a positive signal from the measures of these CSF analytes is "not well defined," concludes that a positive profile of these analytes is "predictive for the evaluation of the ADdementia type" [61]. Importantly, there also have been calls to better exploit the potential of genetics $[62 \cdot \bullet]$ and proteomics [63], and to look beyond $A \beta$ and tau for potential "second hits" that might improve our abilities to predict disease progression. Nevertheless, a recent failure to confirm a plasma biomarker panel for $\mathrm{AD}$ is a sobering reminder of the difficulties inherent in these efforts [64].

SNCA has extensive face validity as a biomarker for PD, with both point mutations and duplication/triplication of the wild-type SNCA locus causing autosomal dominant disease [4], while SNCA deposits in the form of Lewy bodies and Lewy neurites are hallmark pathologies [65]. Just as with tau, SNCA is an intracellular protein whose exocytosis has recently been reported $[66,67]$. Thus, levels in CSF may be influenced by altered active exocytosis and passive release from degenerating neurons. CSF levels of SNCA decline with disease progression, ostensibly due to aggregation of protein $[68,69]$. Both SNCA and tau feature prominently in the Manhattan plots of GWAS studies for PD [70]; however, CSF tau levels are relatively unremarkable in PD and tau does not contribute appreciably to pathology in this disease. Again, similar to tau, SNCA exists in multiple isoforms [71-73], is subject to post-translational modifications, which have been implicated in pathogenesis and which may signal acceleration of the disease process, such as phosphorylation and nitration [74-81] and truncations [82-84], while the most commonly employed assays are agnostic to the presence of these modifications. Nevertheless, there has been a call to qualify SNCA as a biomarker for PD [69]. In contrast to AD biomarker research, which is well established, research in the field of PD biomarkers is both less driven by established convention and less developed than that of $\mathrm{AD}$ biomarkers. Currently, there are significant ongoing efforts to address PD biomarker development in a coherent and comprehensive manner through the efforts of the Parkinson's Progression Markers Initiative (PPMI) as well as other nonprofit and precompetitive initiatives.

\section{Clinical Validation and Regulatory Challenges}

Biomarker discovery and development is a challenging and protracted process. Between 1995 and 2010, the FDAapproved biomarker assays at a rate of fewer than two assays per year [85]. Because primary research continues unabated during the lengthy process of biomarker validation, important post-translational modifications or completely new and potentially more relevant candidate biomarkers may emerge in the interim. Among the difficulties in validating clinical biomarkers are disease heterogeneity, clinical diagnostic accuracy, and the possibility of the coexistence of multiple disease processes [86].

Clinical validation of biomarkers typically occurs in parallel with the development of a drug targeting a particular mechanism of action. Alternatively, biomarkers may be developed to assess the safety or efficacy of several drugs having a common efficacy or toxicity mechanism. The latter instance has led to the FDA Biomarker Qualification Process [87] for such critical end points as kidney toxicity [88, 89] subsequent to the launch of the FDA Critical Path Initiative (CPath [90]). Efforts to enhance precompetitive biomarker development in neurological diseases led CPath, the Brookings Institute, the FDA, EMEA, the NIA, the National Institute of Neurological Disorders and Stroke (NINDS) and multiple industry and academic representatives to form the Coalition Against Major Diseases (CAMD [91]) to develop disease progression models, help identify biomarkers, and help drive collaboration and consensus among investigators and regulatory agencies.

Just as with preclinical biomarkers, the initial step in the qualification of a clinical biomarker remains the context of use, answering the question, "what critical decisions in drug development and the regulatory review will be addressed by this biomarker [92]?" This provides the basis for a gap analysis to drive acquisition of appropriate data sets necessary to support the proposed context of use. Yet, the complexity and uncertainty of these efforts are enormous. Broich and colleagues [93] point out that a plausible hypothesis in basic science is sometimes disproved in the clinic. For example, one would assume that brain atrophy would be positively correlated with progression of dementia, while in the AN1792 A $\beta$ vaccine trial, brain hippocampal volumes decreased in those patients with clinical improvement. FDA review of biomarkers considers the potential risk of such incorrect results (false negative, false positive results) in determining the overall risk assessment of a diagnostic assay: a higher risk assay would be classified 
as class III and would require a premarket application (PMA), while most would be categorized as class II requiring a $510(\mathrm{k})$ submission. The FDA process for a proteinbased biomarker is outlined in a mock presubmission reported by Regnier et al. [94].

\section{Conclusions}

Biomarker discovery and validation are integral components of the drug discovery process, and require clear definition of intended use and rigorous analytical assay validation. While regulatory approval of clinical biomarkers has been slow, a rational approach towards accelerating diagnostics development is currently emerging [95]. This is driven in part by precompetitive consortia and by increasing clarity regarding requirements from regulatory agencies [96], including specific recommendations regarding biomarker qualification [97]. New methods and strategies for biomarker discovery have been reported $[10 \bullet, 12 \bullet, 62 \bullet \cdot$ and may aid in the development of critically needed therapies.

Disclosures No potential conflicts of interest relevant to tis article were reported.

\section{References}

Papers of particular interest, published recently, have been highlighted As:

- Of importance

-• Of major importance

1. Day M, Rutkowski JL, Feuerstein GZ. Translational medicine-a paradigm shift in modern drug discovery and development: the role of biomarkers. Adv Exp Med Biol. 2009;655:1-12.

2. Soares HD. The use of mechanistic biomarkers for evaluating investigational CNS compounds in early drug development. Curr Opin Investig Drugs. 2010;11:795-801.

3. Bekris LM, Yu CE, Bird TD, Tsuang DW. Genetics of Alzheimer disease. J Geriatr Psychiatry Neurol. 2010;23:213-27.

4. Bekris LM, Mata IF, Zabetian CP. The genetics of Parkinson disease. J Geriatr Psychiatry Neurol. 2010;23:228-42.

5. Shi M, Caudle WM, Zhang J. Biomarker discovery in neurodegenerative diseases: a proteomic approach. Neurobiol Dis. 2009;35:157-64.

6. Bahl JM, Jensen SS, Larsen MR, Heegaard NH. Characterization of the human cerebrospinal fluid phosphoproteome by titanium dioxide affinity chromatography and mass spectrometry. Anal Chem. 2008;80:6308-16.

7. Zhang J, Sokal I, Peskind ER, et al. CSF multianalyte profile distinguishes Alzheimer and Parkinson diseases. Am J Clin Pathol. 2008;129:526-9.

8. Calciano MA, Zhou W, Snyder PJ, Einstein R. Drug treatment of Alzheimer's disease patients leads to expression changes in peripheral blood cells. Alzheimers Dement. 2010;6:386-93.
9. Nalls MA, Plagnol V, Hernandez DG, et al. Imputation of sequence variants for identification of genetic risks for Parkinson's disease: a meta-analysis of genome-wide association studies. Lancet. 2011;377:641-9.

10. • Etheridge A, Lee I, Hood L, et al. Extracellular microRNA: a new source of biomarkers. Mutat Res 2011;717:85-90. This paper describes important new biomarker targets and methodologies, which will have a significant impact on future biomarker and drug discovery research.

11. Cogswell JP, Ward J, Taylor IA, et al. Identification of miRNA changes in Alzheimer's disease brain and CSF yields putative biomarkers and insights into disease pathways. J Alzheimers Dis. 2008; 14:27-41.

12. - Fehlbaum-Beurdeley P, Jarrige-Le Prado AC, Pallares D, et al. Toward an Alzheimer's disease diagnosis via high-resolution blood gene expression. Alzheimers Dement 2010;6: 25-38. This paper describes important new biomarker targets and methodologies which will have a significant impact on future biomarker and drug discovery research.

13. Ikemura M, Saito Y, Sengoku R, et al. Lewy body pathology involves cutaneous nerves. J Neuropathol Exp Neurol. 2008;67:94553.

14. Lebouvier T, Neunlist M, Bruley des Varannes S, et al. Colonic biopsies to assess the neuropathology of Parkinson's disease and its relationship with symptoms. PLoS One. 2010;5:e12728.

15. Del Tredici K, Hawkes CH, Ghebremedhin E, Braak H. Lewy pathology in the submandibular gland of individuals with incidental Lewy body disease and sporadic Parkinson's disease. Acta Neuropathol. 2010;119:703-13.

16. Katz R. Biomarkers and surrogate markers: an FDA perspective. NeuroRx. 2004;1:189-95.

17. Waterton JC, Pylkkanen L. Qualification of imaging biomarkers for oncology drug development. Eur J Cancer. 2012;48:409-15.

18. Bansal S, DeStefano A. Key elements of bioanalytical method validation for small molecules. AAPS J. 2007;9:E109-14.

19. Kelley M, DeSilva B. Key elements of bioanalytical method validation for macromolecules. AAPS J. 2007;9:E156-63.

20. Viswanathan CT, Bansal S, Booth B, et al. Quantitative bioanalytical methods validation and implementation: best practices for chromatographic and ligand binding assays. Pharm Res. 2007;24:1962-73.

21. Nowatzke W, Woolf E. Best practices during bioanalytical method validation for the characterization of assay reagents and the evaluation of analyte stability in assay standards, quality controls, and study samples. AAPS J. 2007;9:E117-22.

22. European Medicines Agency: Draft Guideline on Validation of Bioanalytical Methods. Available at www.emea.europa.eu/docs/ en_GB/document_library/Scientific_guideline/2009/12/ WC̄500018062.pdf In Edition 2009.

23. Findlay JW, Smith WC, Lee JW, et al. Validation of immunoassays for bioanalysis: a pharmaceutical industry perspective. J Pharm Biomed Anal. 2000;21:1249-73.

24. Valentin MA, Ma S, Zhao A, et al. Validation of immunoassay for protein biomarkers: bioanalytical study plan implementation to support pre-clinical and clinical studies. J Pharm Biomed Anal. 2011;55:869-77.

25. DeSilva B, Smith W, Weiner R, et al. Recommendations for the bioanalytical method validation of ligand-binding assays to support pharmacokinetic assessments of macromolecules. Pharm Res. 2003;20:1885-900.

26. Rhyne PW, Wong OT, Zhang YJ, Weiner RS. Electrochemiluminescence in bioanalysis. Bioanalysis. 2009;1:919-35.

27. Guglielmo-Viret V, Attree O, Blanco-Gros V, Thullier P. Comparison of electrochemiluminescence assay and ELISA for the detection of Clostridium botulinum type B neurotoxin. J Immunol Methods. 2005;301:164-72. 
28. Mikulskis A, Yeung D, Subramanyam M, Amaravadi L. Solution ELISA as a platform of choice for development of robust, drug tolerant immunogenicity assays in support of drug development. J Immunol Methods. 2011;365:38-49.

29. Eglen RM, Reisine T, Roby $P$, et al. The use of AlphaScreen technology in HTS: current status. Curr Chem Genomics. 2008;1:2-10.

30. Gubala V, Le Guevel X, Nooney R, et al. A comparison of mono and multivalent linkers and their effect on the colloidal stability of nanoparticle and immunoassays performance. Talanta. 2010;81:1833-9.

31. Darmanis S, Nong RY, Hammond M, et al. Sensitive plasma protein analysis by microparticle-based proximity ligation assays. Mol Cell Proteomics. 2010;9:327-35.

32. Glickman JF, Wu X, Mercuri R, et al. A comparison of ALPHAScreen, TR-FRET, and TRF as assay methods for FXR nuclear receptors. J Biomol Screen. 2002;7:3-10.

33. Soderstrom CI, Spriggs FP, Song W, Burrell S. Comparison of four distinct detection platforms using multiple ligand binding assay formats. J Immunol Methods. 2011;371:106-13.

34. Morris JC, Selkoe DJ. Recommendations for the incorporation of biomarkers into Alzheimer clinical trials: an overview. Neurobiol Aging. 2011;32 Suppl 1:S1-3.

35. Gao CM, Yam AY, Wang X, et al. Abeta40 oligomers identified as a potential biomarker for the diagnosis of Alzheimer's disease. PLoS One. 2010;5:e15725.

36. Xia W, Yang T, Shankar G, et al. A specific enzyme-linked immunosorbent assay for measuring beta-amyloid protein oligomers in human plasma and brain tissue of patients with Alzheimer disease. Arch Neurol. 2009;66:190-9.

37. Jawhar S, Wirths O, Bayer TA. Pyroglutamate amyloid-beta (Abeta): a hatchet man in Alzheimer disease. J Biol Chem. 2011;286:38825-32.

38. Bjerke M, Portelius E, Minthon L, et al. Confounding factors influencing amyloid Beta concentration in cerebrospinal fluid. Int J Alzheimers Dis 2010, 2010, Article ID 986310.

39. Lewczuk P, Beck G, Esselmann H, et al. Effect of sample collection tubes on cerebrospinal fluid concentrations of tau proteins and amyloid beta peptides. Clin Chem. 2006;52:332-4.

40. Bateman RJ, Wen G, Morris JC, Holtzman DM. Fluctuations of CSF amyloid-beta levels: implications for a diagnostic and therapeutic biomarker. Neurology. 2007;68:666-9.

41. Vogelgesang S, Jedlitschky G, Brenn A, Walker LC. The role of the ATP-binding cassette transporter P-glycoprotein in the transport of beta-amyloid across the blood-brain barrier. Curr Pharm Des. 2011;17:2778-86.

42. Silverberg GD, Messier AA, Miller MC, et al. Amyloid efflux transporter expression at the blood-brain barrier declines in normal aging. J Neuropathol Exp Neurol. 2010;69:1034-43.

43. Fujiyoshi M, Tachikawa M, Ohtsuki S, et al. Amyloid-beta peptide (1-40) elimination from cerebrospinal fluid involves low-density lipoprotein receptor-related protein 1 at the blood-cerebrospinal fluid barrier. J Neurochem. 2011;118:407-15.

44. Mawuenyega KG, Sigurdson W, Ovod V, et al. Decreased clearance of CNS beta-amyloid in Alzheimer's disease. Science. 2010;330:1774.

45. Buchhave P, Minthon L, Zetterberg H, et al. Cerebrospinal Fluid Levels of beta-Amyloid 1-42, but Not of Tau, Are Fully Changed Already 5 to 10 Years Before the Onset of Alzheimer Dementia. Arch Gen Psychiatry. 2012;69:98-106.

46. Bateman RJ, Munsell LY, Morris JC, et al. Human amyloid-beta synthesis and clearance rates as measured in cerebrospinal fluid in vivo. Nat Med. 2006;12:856-61.

47. Bateman RJ, Siemers ER, Mawuenyega KG, et al. A gammasecretase inhibitor decreases amyloid-beta production in the central nervous system. Ann Neurol. 2009;66:48-54.
48. Imbimbo BP, Peretto I. Semagacestat, a gamma-secretase inhibitor for the potential treatment of Alzheimer's disease. Curr Opin Investig Drugs. 2009;10:721-30.

49. Galimberti D, Scarpini E. Genetics and biology of Alzheimer's disease and frontotemporal lobar degeneration. Int J Clin Exp Med. 2010;3:129-43.

50. Kim W, Lee S, Hall GF. Secretion of human tau fragments resembling CSF-tau in Alzheimer's disease is modulated by the presence of the exon 2 insert. FEBS Lett. 2010;584:3085-8.

51. Yamada K, Cirrito JR, Stewart FR, et al. In vivo microdialysis reveals age-dependent decrease of brain interstitial fluid tau levels in P301S human tau transgenic mice. J Neurosci. 2011;31:13110-7.

52. Kovacech B, Novak M. Tau truncation is a productive posttranslational modification of neurofibrillary degeneration in Alzheimer's disease. Curr Alzheimer Res. 2010;7:708-16.

53. Portelius E, Hansson SF, Tran AJ, et al. Characterization of tau in cerebrospinal fluid using mass spectrometry. J Proteome Res. 2008;7:2114-20.

54. Fagan AM, Shaw LM, Xiong C, et al. Comparison of Analytical Platforms for Cerebrospinal Fluid Measures of \{beta\}-Amyloid 142, Total tau, and P-tau181 for Identifying Alzheimer Disease Amyloid Plaque Pathology. Arch Neurol. 2011;68:1137-44.

55. Verwey NA, van der Flier WM, Blennow K, et al. A worldwide multicentre comparison of assays for cerebrospinal fluid biomarkers in Alzheimer's disease. Ann Clin Biochem. 2009;46:235-40.

56. Mattsson N, Andreasson U, Persson S, et al. The Alzheimer's Association external quality control program for cerebrospinal fluid biomarkers. Alzheimers Dement. 2011;7:386-95. e386.

57. Vanderstichele H, Bibl M, Engelborghs S, et al. Standardization of preanalytical aspects of cerebrospinal fluid biomarker testing for Alzheimer's disease diagnosis: A consensus paper from the Alzheimer's Biomarkers Standardization Initiative. Alzheimers Dement. 2012;8:65-73.

58. Johansson P, Mattsson N, Hansson O, et al. Cerebrospinal fluid biomarkers for Alzheimer's disease: diagnostic performance in a homogeneous mono-center population. J Alzheimers Dis. 2011;24:537-46.

59. Albert MS, DeKosky ST, Dickson D, et al. The diagnosis of mild cognitive impairment due to Alzheimer's disease: recommendations from the National Institute on Aging-Alzheimer's Association workgroups on diagnostic guidelines for Alzheimer's disease. Alzheimers Dement. 2011;7:270-9.

60. McKhann GM, Knopman DS, Chertkow H, et al. The diagnosis of dementia due to Alzheimer's disease: recommendations from the National Institute on Aging-Alzheimer's Association workgroups on diagnostic guidelines for Alzheimer's disease. Alzheimers Dement. 2011;7:263-9.

61. Isaac M, Vamvakas S, Abadie E, et al. Qualification opinion of novel methodologies in the predementia stage of Alzheimer's disease: cerebro-spinal-fluid related biomarkers for drugs affecting amyloid burden-regulatory considerations by European Medicines Agency focusing in improving benefit/risk in regulatory trials. Eur Neuropsychopharmacol. 2011;21:781-8.

62. •• Holtzman DM, Goate A, Kelly J, Sperling R. Mapping the road forward in Alzheimer's disease. Sci Transl Med 2011;3:114ps148. This is an important critical review of current biomarker approaches in neurodegeneration, and a studied proposal for future efforts.

63. Blennow K, Hampel H, Weiner M, Zetterberg H. Cerebrospinal fluid and plasma biomarkers in Alzheimer disease. Nat Rev Neurol. 2010;6:131-44

64. Bjorkqvist M, Ohlsson M, Minthon L, Hansson O. Evaluation of a previously suggested plasma biomarker panel to identify Alzheimer's disease. PLoS One. 2012;7:e29868.

65. Iwatsubo T. Aggregation of alpha-synuclein in the pathogenesis of Parkinson's disease. J Neurol. 2003;250(3):III11-4. 
66. Lee HJ, Patel S, Lee SJ. Intravesicular localization and exocytosis of alpha-synuclein and its aggregates. J Neurosci. 2005;25:601624

67. Liu J, Zhang JP, Shi M, et al. Rab11a and HSP90 regulate recycling of extracellular alpha-synuclein. J Neurosci. 2009;29:1480 5.

68. El-Agnaf OM, Salem SA, Paleologou KE, et al. Alpha-synuclein implicated in Parkinson's disease is present in extracellular biological fluids, including human plasma. FASEB J. 2003;17:1945-7.

69. Mollenhauer B, El-Agnaf OM, Marcus K, et al. Quantification of alpha-synuclein in cerebrospinal fluid as a biomarker candidate: review of the literature and considerations for future studies. Biomark Med. 2010;4:683-99.

70. Edwards TL, Scott WK, Almonte C, et al. Genome-wide association study confirms SNPs in SNCA and the MAPT region as common risk factors for Parkinson disease. Ann Hum Genet. 2010;74:97-109.

71. Beyer K. Alpha-synuclein structure, posttranslational modification and alternative splicing as aggregation enhancers. Acta Neuropathol. 2006;112:237-51.

72. Beyer K, Domingo-Sabat M, Lao JI, et al. Identification and characterization of a new alpha-synuclein isoform and its role in Lewy body diseases. Neurogenetics. 2008;9:15-23.

73. Beyer K, Lao JI, Carrato C, et al. Differential expression of alphasynuclein isoforms in dementia with Lewy bodies. Neuropathol Appl Neurobiol. 2004;30:601-7.

74. Chen L, Periquet M, Wang X, et al. Tyrosine and serine phosphorylation of alpha-synuclein have opposing effects on neurotoxicity and soluble oligomer formation. J Clin Invest. 2009;119:3257-65.

75. Hasegawa M, Fujiwara H, Nonaka T, et al. Phosphorylated alphasynuclein is ubiquitinated in alpha-synucleinopathy lesions. J Biol Chem. 2002;277:49071-6.

76. Inglis KJ, Chereau D, Brigham EF, et al. Polo-like kinase 2 (PLK2) phosphorylates alpha-synuclein at serine 129 in central nervous system. J Biol Chem. 2009;284:2598-602.

77. Mbefo MK, Paleologou KE, Boucharaba A, et al. Phosphorylation of synucleins by members of the Polo-like kinase family. J Biol Chem. 2010;285:2807-22.

78. Paleologou KE, Oueslati A, Shakked G, et al. Phosphorylation at S87 is enhanced in synucleinopathies, inhibits alpha-synuclein oligomerization, and influences synuclein-membrane interactions. J Neurosci. 2010;30:3184-98.

79. Duda JE, Giasson BI, Chen Q, et al. Widespread nitration of pathological inclusions in neurodegenerative synucleinopathies. Am J Pathol. 2000;157:1439-45.

80. Giasson BI, Duda JE, Murray IV, et al. Oxidative damage linked to neurodegeneration by selective alpha-synuclein nitration in synucleinopathy lesions. Science. 2000;290:985-9.

81. Souza JM, Giasson BI, Chen Q, et al. Dityrosine cross-linking promotes formation of stable alpha -synuclein polymers. Implication of nitrative and oxidative stress in the pathogenesis of neurodegenerative synucleinopathies. J Biol Chem. 2000;275:18344-9.

82. Hoyer W, Cherny D, Subramaniam V, Jovin TM. Impact of the acidic C-terminal region comprising amino acids $109-140$ on alphasynuclein aggregation in vitro. Biochemistry. 2004;43:16233-42.
83. Li W, West N, Colla E, et al. Aggregation promoting C-terminal truncation of alpha-synuclein is a normal cellular process and is enhanced by the familial Parkinson's disease-linked mutations. Proc Natl Acad Sci U S A. 2005;102:2162-7.

84. Oueslati A, Fournier M, Lashuel HA. Role of post-translational modifications in modulating the structure, function and toxicity of alpha-synuclein: implications for Parkinson's disease pathogenesis and therapies. Prog Brain Res. 2010;183:115-45.

85. Anderson NL. The clinical plasma proteome: a survey of clinical assays for proteins in plasma and serum. Clin Chem. 2010;56:17785.

86. Nyhlen J, Constantinescu R, Zetterberg H. Problems associated with fluid biomarkers for Parkinson's disease. Biomark Med. 2010;4:671-81.

87. Goodsaid F, Frueh F. Biomarker qualification pilot process at the US Food and Drug Administration. AAPS J. 2007;9:E105-8.

88. Goodsaid FM, Mendrick DL. Translational medicine and the value of biomarker qualification. Sci Transl Med. 2010;2:47ps44.

89. Dieterle F, Sistare F, Goodsaid F, et al. Renal biomarker qualification submission: a dialog between the FDA-EMEA and Predictive Safety Testing Consortium. Nat Biotechnol. 2010;28:455-62.

90. Woodcock J, Woosley R. The FDA critical path initiative and its influence on new drug development. Annu Rev Med. 2008;59:112.

91. Romero K, de Mars M, Frank D, et al. The coalition against major diseases: Developing tools for an integrated drug development process for Alzheimer's and Parkinson's diseases. Clin Pharmacol Ther. 2009;86:365-7.

92. Goodsaid F, Papaluca M. Evolution of biomarker qualification at the health authorities. Nat Biotechnol. 2010;28:441-3.

93. Broich K, Weiergraber M, Hampel H. Biomarkers in clinical trials for neurodegenerative diseases: regulatory perspectives and requirements. Prog Neurobiol. 2011;95:498-500.

94. Regnier FE, Skates SJ, Mesri M, et al. Protein-based multiplex assays: mock presubmissions to the US Food and Drug Administration. Clin Chem. 2010;56:165-71.

95. Anderson L. Within sight of a rational pipeline for development of protein diagnostics. Clin Chem. 2012;58:28-30.

96. Scherf U, Becker R, Chan M, Hojvat S. Approval of novel biomarkers: FDA's perspective and major requests. Scand J Clin Lab Invest Suppl. 2010;242:96-102.

97. Food and Drug Administration: Guidance for Industry, E16: Biomarkers Related to Drug or Biotechnology Product Development: Context, Structure, and Format of Qualification Submissions. Available at www.fda.gov/downloads/Drugs/ GuidanceComplianceRegulatoryInformation/Guidances/ UCM267449.pdf. In Edition 2011.

98. National Institutes of Health Center for Translational Therapeutics (NCTT) Assay Guidance Section2: Plate Uniformity and Signal Variability Assessment. Available at: http://assay.nih.gov/assay/ index.php/Section2:Plate Uniformity and Signal Variability Assessment\#No_drift_or_edge_effects

99. Frasier M, Chowdhury S, Eberling J, Sherer T. Biomarkers in Parkinson's disease: a funder's perspective. Biomarkers Med. 2010;4:723-9. 